\title{
RECENT PROGRESS IN POWER REFRIGERATION BELOW 2 K FOR SUPERCONDUCTING ACCELERATORS
}

\author{
S. Claudet
}

As a result of technico-economical optimization and quest for increased performance, $2 \mathrm{~K}$ cryogenics is now present in large accelerator projects using superconducting magnets or acceleration cavities. Consequently, large cryogenic systems producing refrigeration capacity below $2 \mathrm{~K}$ in the $\mathrm{kW}$ range and with high efficiency over a large dynamic range are needed. After CEBAF and SNS, this is the case for the Large Hadron Collider (LHC) project at CERN for which eight $2.4 \mathrm{~kW} @ 1.8 \mathrm{~K}$ refrigeration units are needed to cool each a $3.3 \mathrm{~km}$ long sector of high-field magnets. Combining cold hydrodynamic compressors in series with warm volumetric compressors, complete pre-series units as well as sets of series cold compressors have been intensively tested and validated from two different industrial suppliers.

After recalling the possible $2 \mathrm{~K}$ refrigeration cycles and their comparative merits, this paper describes the specific features of the LHC system and presents the achieved performance with emphasis on the progress in terms of efficiency, operational compliance, reliability and maintenance. Perspectives of application to a future International Linear Collider (ILC) based on cold RF technology are then briefly evoked. 


\title{
RECENT PROGRESS IN POWER REFRIGERATION BELOW $2 \mathrm{~K}$ FOR SUPERCONDUCTING ACCELERATORS
}

\author{
S. Claudet, CERN, Geneva, Switzerland
}

\begin{abstract}
As a result of technico-economical optimization and quest for increased performance, $2 \mathrm{~K}$ cryogenics is now present in large accelerator projects using superconducting magnets or acceleration cavities. Consequently, large cryogenic systems producing refrigeration capacity below $2 \mathrm{~K}$ in the $\mathrm{kW}$ range and with high efficiency over a large dynamic range are needed.

After CEBAF and SNS, this is the case for the Large Hadron Collider (LHC) project at CERN for which eight $2.4 \mathrm{~kW} @ 1.8 \mathrm{~K}$ refrigeration units are needed to cool each a $3.3 \mathrm{~km}$ long sector of high-field magnets. Combining cold hydrodynamic compressors in series with warm volumetric compressors, complete pre-series units as well as sets of series cold compressors have been intensively tested and validated from two different industrial suppliers.

After recalling the possible $2 \mathrm{~K}$ refrigeration cycles and their comparative merits, this paper describes the specific features of the LHC system and presents the achieved performance with emphasis on the progress in terms of efficiency, operational compliance, reliability and maintenance. Perspectives of application to a future International Linear Collider (ILC) based on cold RF technology are then briefly evoked.
\end{abstract}

\section{INTRODUCTION}

Superfluid cryogenics is now widely present in large accelerator projects using applied superconductivity [1]. In the 1980's, Tore Supra (TS) [2] was the first large physics instrument using a cryogenic system at $1.8 \mathrm{~K}$ and has demonstrated the technical feasibility of such systems. CEBAF [3] was the first accelerator project using this technology for cooling superconducting $\mathrm{RF}$ cavities, to be followed soon by SNS [4]. The LHC project [5] makes use of large cryogenic capacity at $1.8 \mathrm{~K}$ for cooling superconducting high-field magnets. Future accelerator projects like TESLA [6] or the ILC are also based on superfluid technology. Table 1 gives the main characteristics of superfluid cryogenic systems. Some existing $4.5 \mathrm{~K}$ projects like the LHD [7] have already designed their cooling schemes for an eventual $1.8 \mathrm{~K}$ upgrade.

Table 1: Main superfluid cryogenic systems

\begin{tabular}{|l|c|c|c|c|}
\cline { 2 - 5 } \multicolumn{1}{c|}{} & TS & $\begin{array}{c}\text { CEBAF } \\
\text { - SNS }\end{array}$ & LHC & TESLA \\
\hline Temperature [K] & 1.8 & 2 & 1.9 & 2 \\
\hline Nb of units [-] & 1 & $2-1$ & 8 & 7 \\
\hline Capacity/unit [W] & 300 & 4800 & 2400 & 4250 \\
\hline Length/sector [m] & N/A & 500 & 3300 & 2500 \\
\hline
\end{tabular}

The cost optimisation of accelerators impacts on their choice of operating temperature. In the case of high frequency superconducting acceleration cavities, the main drive for superfluid cooling is the exponential dependence of the BCS losses on the ratio of operating-to-critical temperature. Accelerators based on this technology operate around $2 \mathrm{~K}$ to minimise the capital costs and overall energy consumption. In the case of high-field superconducting cryomagnets, the main drive for superfluid cooling is the decrease of the critical current density of the superconducting material with field, and particularly the need of increasing the current carrying capacity of NbTi. This also minimise the amount of superconductor and hence the capital costs.

\section{POSSIBLE THERMODYNAMIC CYCLES}

The saturation pressure of helium at $2 \mathrm{~K}$ is $3 \mathrm{kPa}$ : all refrigeration cycles below $2 \mathrm{~K}$ must therefore perform compression with a minimum pressure ratio of about 40 to bring the process fluid back to atmospheric.

Two types of cycles are generally considered to produce refrigeration below $2 \mathrm{~K}$ (see generic scheme in Figure 1):

- The "integral-cold" compression cycle based on multistage cold compressors (CC).

- The "mixed" compression cycle based on a combination of cold compressors in series with warm sub-atmospheric compressors.

In advanced accelerators, the dynamic heat loads correspond to a large part of the total load. Between standby operating mode without beams and full-load operating mode, large dynamic ranges (factor 3 for LHC and up to 7 for TESLA) are required for the superfluid cryogenic system. The choice of cycle must therefore take all these aspects into account.

\section{Integral Cold Compression Cycles}

For operating temperature between $2 \mathrm{~K}$ and $1.8 \mathrm{~K}$, the "integral cold" compression cycle requires at least 4 stages in series in order to perform the overall pressure ratio of 40 to 80 . The compressed helium is directly reinjected in the cold low-pressure (LP) stream of the $4.5 \mathrm{~K}$ refrigerator.

The main drawback of this cycle concerns the turndown capability. The cold compressor set has to guarantee the same pressure ratio for any load. Due to the limited operating range of hydrodynamic machines working at constant pressure ratio, only a reduction of $20 \%$ of the flow rate is possible, i.e. a dynamic range limited to a factor 1.25. Below this limit, additional electrical heating has to be introduced to compensate for 
the load reduction. An possible alternative to be further developed would consist in the introduction of a fifth cold compressor in series with the 4 mentioned before, with the aim of reaching a dynamic range of 2 . Such a cycle is therefore not very compliant with respect to turndown capability. Consequently, its operating cost is not optimised for low-load operation [8].

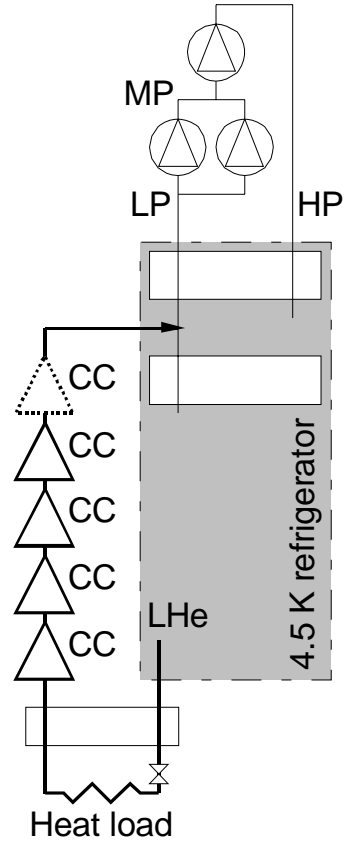

"Integral cold" cycle

\section{Sub-atmospheric compressor MP}

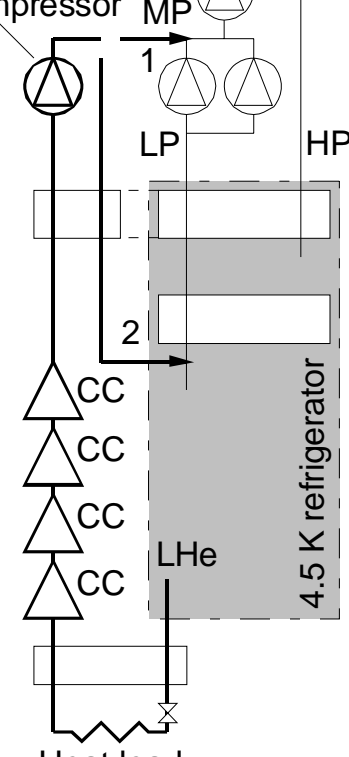

"Mixed" cycle

Figure 1: Generic scheme of $<2 \mathrm{~K}$ refrigeration cycles

\section{"Mixed" Compression Cycles}

Historically, the number of cold compressors was kept as low as possible. With experience and improved performance, up to 4 in series can be envisaged. The compressed helium can be re-injected in the $4.5 \mathrm{~K}$ refrigerator at different levels:

- at the warm medium-pressure (MP) side (connection \#1 on Figure 1). This requires the use of screw compressors having a sufficient built-in pressure ratio to operate sub-atmospheric. In this case, the enthalpy of the gas at the outlet of the cold compressors has to be recovered by the heat exchangers of the $4.5 \mathrm{~K}$ refrigerator. The main advantage of this solution is that, the same oil-removal and final-cleaning systems can be used for the warm sub-atmospheric compressors and for the booster stages of the $4.5 \mathrm{~K}$ refrigerators, minimising the investment cost of the system.

- at the cold low-pressure side (connection\#2 on Figure 1). This is required when the enthalpy of the cold gas at the outlet of the cold compressors cannot be recovered by the heat exchangers of the $4.5 \mathrm{~K}$ refrigerators (LHC case) [9]. In this case, the warm subatmospheric compressor stage requires its own oil removal system and final cleaning system (coalescers and charcoal adsorbers), increasing the investment cost of the system.

The main advantage of the "mixed" cycle resides in its turndown capability. With sub-atmospheric warm compressors having volumetric characteristics, the pressure at the outlet of the cold compressors decreases linearly with respect to the flow-rate to be compressed. Such a cycle can accommodate a large dynamic range without any additional electrical heating. In addition, the total pressure ratio to be performed by the cold compressors is reduced and the speed of some machines can be lowered, thus decreasing the total compression power and saving operating cost.

In addition, the warm sub-atmospheric compressors are very useful during transient operation modes like cool-down and pump-down, in which the cold compressors are far from their design conditions and difficult to tune.

The only drawback of this cycle concerns the risk of air inleaks due to the presence of sub-atmospheric circuits which are not under insulation vacuum. Helium guards are recommended to prevent helium pollution.

\section{LHC $1.8 \mathrm{~K}$ SYSTEM}

In 1993, CERN has started a R\&D programme on cold compressors, procuring from European and Japanese companies, three hydrodynamic compressors with the aim to investigate critical issues such as drive and bearing technology, impeller and diffuser hydrodynamics, mechanical and thermal design, as well as their impact on overall efficiency [9].

In parallel and in order to make the best use of the 4 existing $4.5 \mathrm{~K}$ refrigerators from LEP, thorough process analysis was conducted to match the corresponding constraints. A reference cycle was identified [10], based on "mixed" compression with return to the $4.5 \mathrm{~K}$ refrigerator at $20 \mathrm{~K}$.

Following these programmes, the $2.4 \mathrm{~kW}$ at $1.8 \mathrm{~K}$ refrigeration units have been specified in 1998 [11] and four units ordered in 1999 to IHI-Linde (JapanSwitzerland) and Air Liquide (France) [12,13].

In order to validate the process and components, a first "pre-series unit" had to be validated by extensive testing in a dedicated test facility [14] at CERN before launching production of series units. Acceptance tests were performed in 2002 and 2003 in order to measure and validate the overall and detailed performance of the preseries units, and in 2003 and 2004 for series cold compressor.

The key components and most significant performance achieved are presented.

\section{Cold Compressors}

Modern cold compressors features electrical motor drives (3-phase induction motors) with active magnetic bearings working at room temperature and controlled with modern digital electronics, axial-centrifugal (threedimensional) impellers and fixed-vane diffusers, as shown on figure 2 . They are of cartridge type and can be exchanged without breaking the cold box vacuum, 
providing replacement possibility while keeping the accelerator at low temperature.

Figure 3 show a typical operating field of these hydrodynamic compressors, which displays the pressure ratio as a function of the reduced mass-flow $\mathrm{Mr}$ and the reduced speed $\mathrm{Nr}$ defined as follows:

$$
\mathrm{Mr}=\frac{\mathrm{m}}{\mathrm{m}_{0}} \cdot \sqrt{\frac{\operatorname{Tin}_{\operatorname{Tin}_{0}}}{\operatorname{Pin}_{0}}} \frac{\mathrm{Pin}_{\mathrm{P}}}{\mathrm{Nr}}=\frac{\mathrm{N}}{\mathrm{N}_{0}} \cdot \sqrt{\frac{\operatorname{Tin}_{0}}{\operatorname{Tin}}}
$$

with $\mathrm{m}$ the mass-flow, $\mathrm{N}$ the rotational speed, Tin and Pin the inlet temperature and pressure of the cold compressor and with the subscript 0 corresponding to the design conditions.

The working area is limited on the left side by the surge line, on the right by the choke line and on the top by the maximum rotational speed of the drive. At constant pressure ratio, such hydrodynamic machines have a operating margin of 20 to $25 \%$ before reaching the surge line.

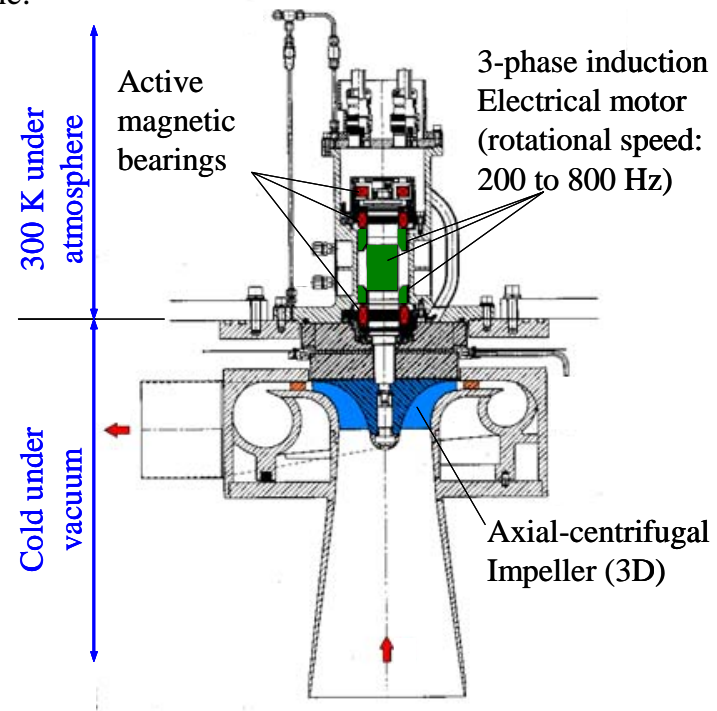

Figure 2: Typical cross section of a cold compressor

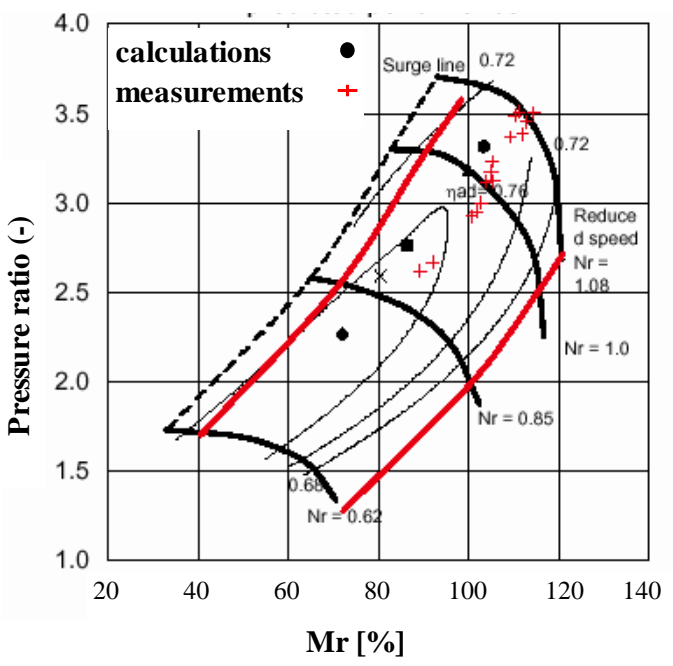

Figure 3: Typical operating field of a cold compressor
Figure 4 summarises the test results at maximum capacity for the cold compressors from each supplier. Reproduce-ability between sets of cartridges has been found within $+/-2 \%$. In most of the operation points and for most of the cold compressors, the isentropic efficiency are above $75 \%$. Efficiency remains above $70 \%$ for values of pressure ratio above 1.5. Maximum pressure ratio of 3.6 were obtained as nominal value, and up to 4.1 in offdesign stable operation.

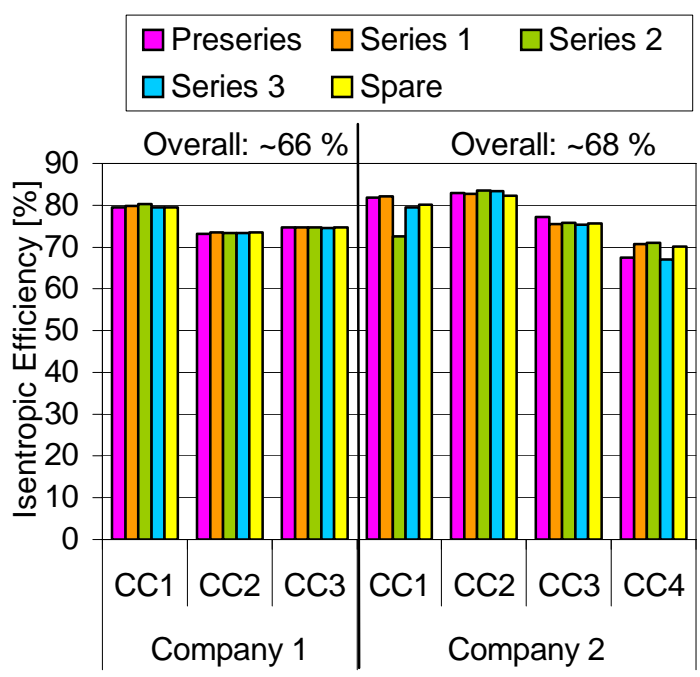

Figure 4: Measured isentropic efficiency

\section{Warm sub-atmospheric Compressors}

Oil lubricated screw compressors (LSC) are positive displacement machines with volumetric characteristics. This type of compressor is the industrial standard used in conventional helium refrigeration. They are proposed as single stage machines, with optimum pressure ratio between 4 and 8 or as compound machines (two in one) with optimum pressure ratio between 15 and 20. Figures $5 \mathrm{a}$ and $5 \mathrm{~b}$ shows the measured isothermal efficiency. The decrease of efficiency with suction pressure is less sensitive than expected. Above 0.25 bar (25 kPa), isothermal efficiency is higher than $42 \%$, to be compared with the 50 to $55 \%$ for standard $4.5 \mathrm{~K}$ helium refrigeration.

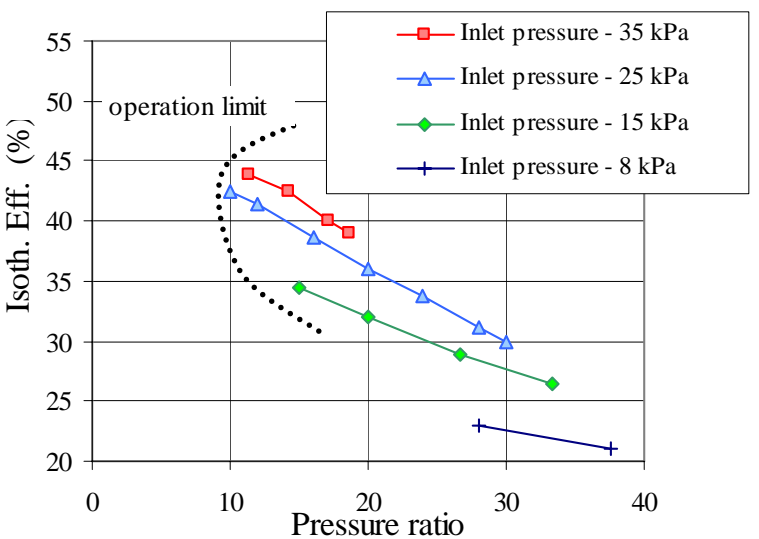

Figure 5a: Isothermal efficiency, single stage LSC 


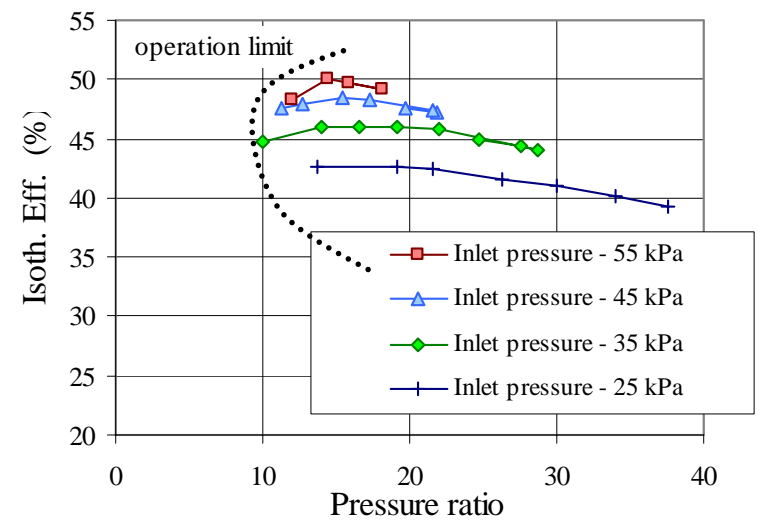

Figure 5b: Isothermal efficiency, 2 stage LSC

\section{Global cycle performance}

To illustrate the thermodynamic efficiency, it is usual to calculate the coefficient of performance (COP) which is the ratio of overall power consumption over the cooling power produced. For the equivalent $4.5 \mathrm{~K}$ load induced by the $1.8 \mathrm{~K}$ units, effective COP were considered [15]. Figure 6 shows the achieved COP. This clearly illustrates the merit of "mixed" cycles for turndown.

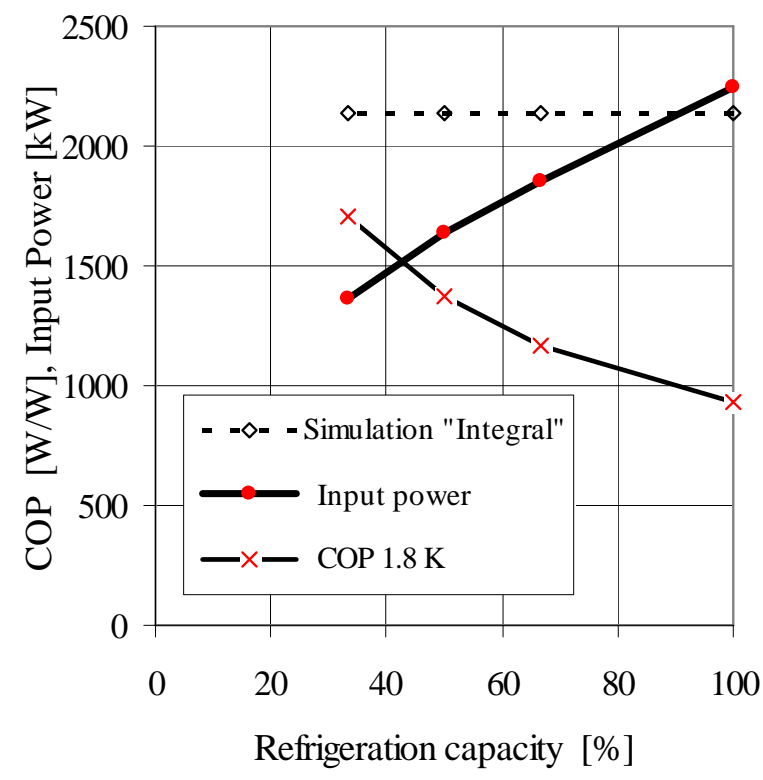

Figure 6: Efficiency at variable load

\section{Additional features}

The only drawback of cycles with sub-atmospheric circuits which are not under insulation vacuum is the risk of pollution due to air inleaks. Welded joints are used whenever possible and helium guards are recommended for remaining cases.

Being able to reach a correct steady-state mode with a chain of hydrodynamic machines is not sufficient: one must also have reliable and mastered pump-down or capacity adaptation. This requires to perform cool-down and pump-down, when the cold compressors are far from their design conditions and difficult to tune. At constant suction pressure and for a given load or mass-flow, the only free parameter to accommodate the cold compressor characteristic is the inlet temperature. This inlet temperature is adjusted by flow mixing: injection of warm gas allows to raise the temperature and a built-in phase separator equipped with electrical heater can generate mass-flow at lower temperature. Therefore, a temperature mixer at the inlet of the first cold compressor is implemented.

To be coherent with common practice for expander turbines, the exchange of cold compressor cartridge was requested in less than 5 hours. It turned out to be the case, with additional features for magnetic bearings. Reduced fabrication and mounting tolerances combined with thorough factory tuning, of all cartridges allows the replacement of a cold compressor cartridge without retuning of magnetic bearings.

\section{PERSPECTIVES FOR FUTURE APPLICATIONS}

Since 2004, the international community has selected the low-temperature superconducting technology for the International Linear Collider (ILC). Based on recent experience, a brief review of parameters or components allowing to minimise investments and operating costs is proposed.

\section{Largest size of refrigerator}

The capital cost of a unit of refrigeration decreases with increasing size of the refrigerator [16]. In addition, larger refrigerators can feed longer cryogenic strings of cryomodules, reducing the total number of cryogenic units necessary. This would result in additional savings in civil engineering and infrastructure.

The maximum size of a $2 \mathrm{~K}$ refrigerator is driven by several factors. One of the most important is the maximum flow achievable by the cold compressors, which is linked to the size of the impeller and maximum tolerated speed. A direct scaling of what is implemented at LHC would allow $250 \mathrm{~g} / \mathrm{s}$ to be processed by one single $2 \mathrm{~K}$ refrigerator, i.e. about $5.2 \mathrm{~kW}$ at $2 \mathrm{~K}$.

\section{Cold compressors}

Their performance has a direct impact on costs. Large individual pressure ratio would allow reducing the number of stages. Present impellers, electrical motor drives and active magnetic bearings would allow pressure ratio of 3.5 to 4.0 for lower stages and 3.0 for higher stages. This tends to reduce the investment cost.

Isentropic efficiencies of $80 \%$ for the lower stages to $75 \%$ for the higher stages would provide a relatively low outlet temperature of the CC train, diminishing the corresponding load on the main $4.5 \mathrm{~K}$ refrigerator. This tends to reduce the operating cost. 


\section{Cycle efficiency and dynamic range}

Experience with cold compressors and continuous improvements in efficiency favour the use of cold compressors all the way up to atmospheric pressure. However, the "mixed" compression cycle with its volumetric warm compressor appears to be better suited for large dynamic range.

For the ILC, an arrangement of 4 cold compressors in series with a screw compressor ("mixed" cycle, connection 1) appears to combine optimal investment and operating costs. Based on proposal for TESLA refrigerator cycle [6], a total pressure ratio to be performed by cold compressors around 40 results in suction pressure of screw compressor slightly above atmospheric pressure. At maximum capacity, this would give a coefficient of performance around $720 \mathrm{~W} / \mathrm{W}$ or $21 \%$ of Carnot.

A dynamic range of 7 is required. The volumetric characteristic and maximum possible pressure ratio of the screw compressor would inherently provide around a factor of 6 . From the dynamic range of 3 achieved for LHC and operational experience so far with adjustments of pressure ratio for each individual stage, we do not see serious reasons that would prevent from reaching the desired factor of 7 .

To cope with transients, the adjustment of inlet temperature by flow mixing is highly recommended.

\section{CONCLUSION}

Superfluid cryogenic systems are now present and foreseen in large projects using applied superconductivity. This is presently the case for LHC, with the $1.8 \mathrm{~K}$ system fulfilling its requirements. The "mixed" compression cycle based on cold compressors in series with warm sub-atmospheric compressors of volumetric type reached the specified maximum capacity and proved to allow at least a dynamic range of 3. The isentropic efficiencies of cold compressors now reach $80 \%$, with achieved pressure ratio above 3.5.

Seven of the eight $1.8 \mathrm{~K}$ units for LHC are now delivered, Two have already demonstrated the specified capacity and global performance. Completion of total acceptance tests is expected early 2006.

With the LHC project, at least two experienced industrial suppliers exist worldwide. New standards for technology, efficiency and operational compliance have been set, providing interesting perspectives for future possible applications.

\section{ACKNOWLEDGEMENTS}

The author would like to thank Ph. Lebrun and L. Tavian for their stimulating encouragements all along these years and for their support to this paper. Thanks to all colleagues and industrial partners involved in defining, building-up and operating these $1.8 \mathrm{~K}$ units at CERN.

\section{REFERENCES}

[1] L. Tavian, "Large cryogenic systems at $1.8 \mathrm{~K}$ ", invited paper presented at EPAC, Vienna, Austria (2000)

[2] G. Claudet et al., "Tore Supra and HeII cooling of large high-field magnet”, Adv. Cryo. Eng. 35A (1990) 55-67

[3] C.H. Rode, “CEBAF cryogenic system”, paper presented at PAC'95, Dallas (1995)

[4] D. Arenius et al.,"Cryogenic system for spallation neutron source (SNS)", Paper presented at CEC'03, Anchorage (2003)

[5] "LHC design report Vol. 1: the LHC main ring", CERN-2004-003-V1

[6] S. Wolff, "The cryogenic system of Tesla”, Tesla 2001-37 (2001)

[7] T. Mito et al., "Development of a cryogenic system for the Large Helicoidal Device”, Adv. Cryo. Eng. 43A (1998) 589-596

[8] S. Claudet et al., "Towards cost-to-performance optimisation of large superfluid helium refrigeration systems”, Proc. ICEC18, Mumbai India (2000) 203206

[9] A. Bézaguet et al., "Performance assessment of industrial prototype cryogenic helium compressors for the Large Hadron Collider", Proc. ICEC17, IoP, Bristol, UK (1998) 148-148

[10] F. Millet et al., “A possible $1.8 \mathrm{~K}$ refrigeration cycle for the Large Hadron Collider”, Adv. Cryo. Eng. 43A (1998) 387-393

[11] S. Claudet et al., "Specification of eight 2400 W @ $1.8 \mathrm{~K}$ refrigeration units for the LHC", Proc. ICEC18, Mumbai India (2000) 207-210

[12] H. Asakura et al., "Four 2400 W / 1.8 K refrigeration units for CERN-LHC: the IHI/Linde system”, Proc. ICEC18, Mumbai India (2000) 215218

[13] B. Hilbert et al., " $2.4 \mathrm{~kW}$ at $1.8 \mathrm{~K}$ refrigeration units for CERN LHC project supplied by Air Liquide”, Proc. ICEC18, Mumbai India (2000) 211-214

[14] S. Claudet et al., "A cryogenic test station for the pre-series 2400 W @1.8 K refrigeration units for the LHC”, Proc. ICEC19, Grenoble, France (2002) 83-86

[15] H. Gruehagen \& U. Wagner, "Measured performance of four $18 \mathrm{~kW}$ @ $4.5 \mathrm{~K}$ helium refrigerators for the LHC cryogenic system”, Proc. ICEC20, Beijing, China (2004)

[16] S. Claudet et al., "Economics of large helium cryogenic system: experience from recent projects at CERN”, Adv. Cryo. Eng. 45B (2000) 1301-1308 